\title{
Peculiarity of stellar kinematics in the solar vicinity
}

\author{
M.E. Popova ${ }^{\star}$ \\ Astronomical Observatory, Ural Federal University, 51 Lenin Street, Yekaterinburg 620000, Russia
}

Received 2013 Apr 3, accepted 2013 Jun 3

Published online 2013 Oct 1

Key words open clusters and associations: general - solar neighborhood - stars: kinematics and dynamics

The peculiar velocities of nearby stars are studied. We determine the solar velocity components from the radial velocities and proper motions of stars and open clusters. We show that nearby stars are not very suitable for the determination of the solar motion components because of peculiarities in their velocity distribution.

\section{Introduction and sample}

The velocity of solar motion is one of the key parameters of stellar kinematics. This parameter is very important for evaluating the kinematical properties of various structural components of the Galaxy. However, there are still significant discrepancies in the determination of solar velocity components (especially in the direction of galactic rotation). For example, Dehnen \& Binney (1998) estimated the solar motion components in the commonly used coordinate system to be $\left(U_{0}, V_{0}, W_{0}\right)=(10.0 \pm 0.4,5.2 \pm 0.6,7.2 \pm 0.4)$ $\mathrm{km} \mathrm{s}^{-1}$, whereas Fehrenbach et al. (2001) obtained a different value, $\left(U_{0}, V_{0}, W_{0}\right)=(2.9 \pm 0.6,10.4 \pm 0.6,4.8 \pm 1.2)$ $\mathrm{km} \mathrm{s}^{-1}$.

The aim of this paper is to determine the components of solar velocity from radial velocities and proper motions of stars and open clusters (OCls) without relying on the immediate solar neighbourhood.

To this end, we use the proper motion data from the Hipparcos catalogue (van Leeuwen, 2007) and the the radial velocity data from the catalogue of Kharchenko et al. (2007) for stars. We use the catalogue of Dias et al. (2002) for OCls.

We use a sample of stars and OCls with heliocentric distances no greater than $300 \mathrm{pc}$ and $1 \mathrm{kpc}$, respectively, and with fractional errors of parallaxes, proper motions and radial velocities not exceeding $30 \%$.

\section{Determination of the solar velocity components from the data for stars}

We subdivided all stars of the sample into six subsamples: subgiants (4190 stars), red giants (1454 stars), and four main-sequence subsamples: MS1 $(B-V \leq 0.2)$ with 2475 stars, MS2 $(0.2<B-V \leq 0.5)$ with 5380 stars, MS3 $(0.5<B-V \leq 0.8)$ with 7736 stars, and MS4 ( $B-V>0.8$ ) with 1567 stars).

\footnotetext{
^ Corresponding author: Maria.Popova@usu.ru
}

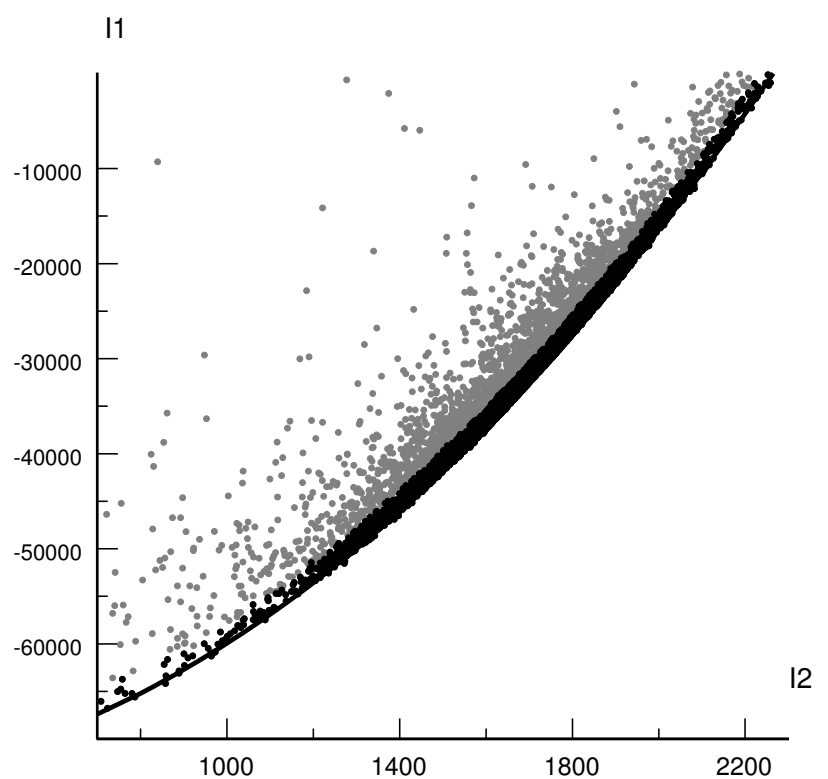

Fig. 1 Selection of stars using the Lindblad diagram (the grey and black dots show all stars and selected subsample stars, respectively).

Stars with low-eccentricity galactic orbits are better representative of the kinematical properties of the thin disc. To select such stars, we used the Lindblad diagram (Fig. 1), where $I 1$ and $I 2$ are the integrals of energy and momentum, respectively. We excluded high-eccentricity stars from our subsamples. We chose the optimum number of stars by setting the eccentricity cutoff so as to minimize the errors of the inferred solar velocity components. Figure 1 shows the Lindblad diagram for stars of MS3 group.

Figure 2 shows an example of the inferred solar velocity components for the central part of the main sequence. Table 1 gives the solar velocity components determined from the radial velocities and proper motions of all subsample stars. 
Table 1 Components of the solar velocity.

\begin{tabular}{lcccc}
\hline Subsamples & $U_{0}\left(\mathrm{~km} \mathrm{~s}^{-1}\right)$ & $V_{0}\left(\mathrm{~km} \mathrm{~s}^{-1}\right)$ & $W_{0}\left(\mathrm{~km} \mathrm{~s}^{-1}\right)$ & Number of selected stars \\
\hline Subgiants & $10.7 \pm 1.9$ & $13.1 \pm 2.2$ & $6.9 \pm 0.6$ & 3129 \\
Red giants & $9.5 \pm 0.8$ & $17.0 \pm 2.7$ & $5.1 \pm 1.2$ & 1054 \\
MS 1 & $11.0 \pm 1.5$ & $15.1 \pm 1.2$ & $7.9 \pm 0.6$ & 1391 \\
MS 2 & $11.8 \pm 0.9$ & $10.6 \pm 1.2$ & $7.6 \pm 0.4$ & 3799 \\
MS 3 & $10.6 \pm 1.4$ & $15.5 \pm 2.0$ & $6.9 \pm 0.5$ & 5519 \\
MS 4 & $14.1 \pm 1.6$ & $19.1 \pm 1.0$ & $6.5 \pm 1.3$ & 1015 \\
Average & $11.1 \pm 0.6$ & $15.1 \pm 1.3$ & $7.1 \pm 0.3$ & \\
\hline
\end{tabular}

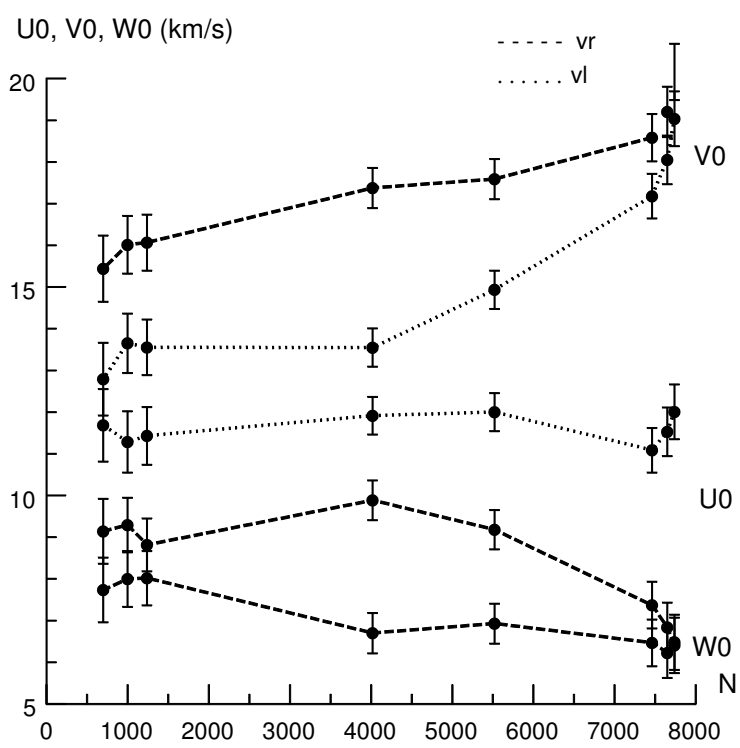

Fig. 2 Solar velocity components determined from the radial velocities and proper motions of stars of MS3 group.

We conclude that selection of stars by the eccentricities of their galactic orbits does not reliably suppress the Stromberg asymmetry in subsamples (especially for the $V_{0}$ component). We believe this to be due to the fact that the adopted method assumes that the distribution of peculiar velocities is free from local features. In reality, the distribution of stellar velocities is highly structured. Large errors (especially in $V_{0}$ ) are therefore due to local effects in the distribution of peculiar velocities of stars. Hence objects should be selected within a greater volume of the Galaxy, and open clusters are very convenient tracers for this purpose especially given that most of them are quite young and move in almost circular orbits.

\section{The determination of the solar velocity components from OCls data}

As is evident from Fig. 3, the peculiar velocities field of OCLs is quite smooth because of the large space volume they sample and because the $\mathrm{OCl}$ sample contains practically no identifiable kinematical groups. Open clusters are

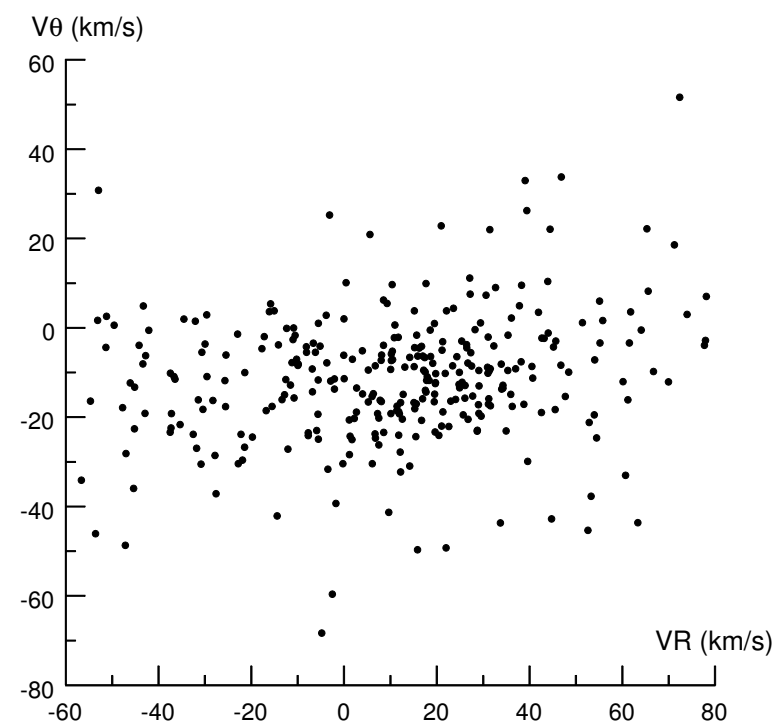

Fig. 3 Peculiar velocities field of OCls. Hereafter $V_{R}>0$ and and $V_{\Theta}>0$ in the direction of galactic anticentre and galactic rotation, respectively.

therefore very convenient objects for the determination of the solar velocity components.

Figure 4 shows the distribution of OCls and their peculiar velocities projected on the galactic plane. We use only OCls with $r \leq 1 \mathrm{kpc}$ to estimate the solar velocity components, because UCAC-2 and UCAC-3 proper motions of faint stars are likely to be fraught with significant systematic errors. OCls with large eccentricities, which are mostly due to large proper-motion errors, were removed. A selection performed using the Lindblad diagram leaves us with a total of 220 OCls.

Figure 5 shows the components of solar velocity $\left(U_{0}\right.$ and $V_{0}$ ) determined from the radial velocities and proper motions of OCls. Because of the low galactic latitudes of OCls we adopt the $W_{0}$ component determined from the data for stars. The resulting solar velocity components based on OCls data are $U_{0}=9.4 \pm 0.2 \mathrm{~km} \mathrm{~s}^{-1}, V_{0}=11.0 \pm 0.5$ $\mathrm{km} \mathrm{s}^{-1}$. 


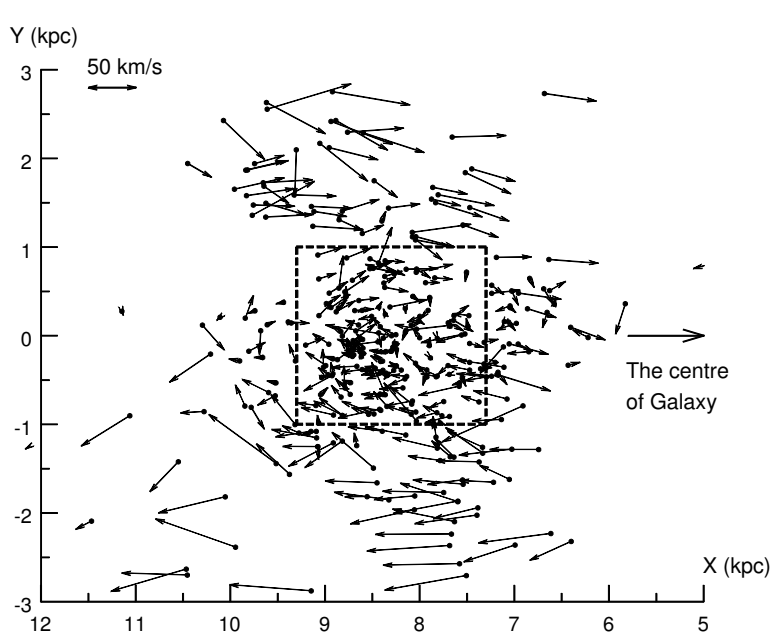

Fig. 4 Distribution of OCls and their peculiar velocities projected on the galactic plane (The Sun is at $X=8.3 \mathrm{kpc}, Y=0.0$ $\mathrm{kpc})$.

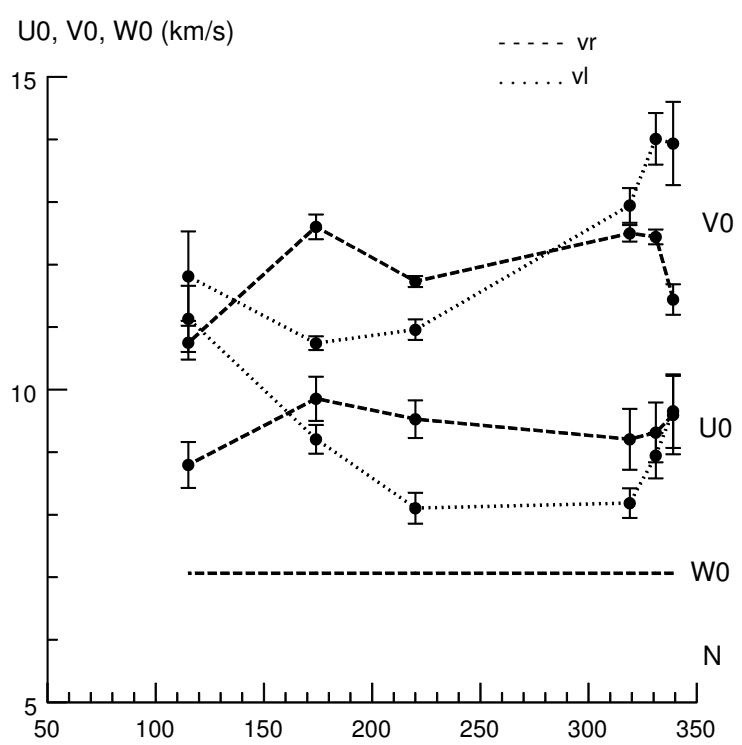

Fig. 5 Components of solar velocity $\left(U_{0}\right.$ and $\left.V_{0}\right)$ determined from the radial velocities and proper motions of OCls.

\section{The peculiar velocities field of the stars}

The peculiar velocities field of stars is not smooth, unlike that of OCls, where kinematical groups are difficult to identify (see Fig. 6a). By applying the smoothing procedure to the velocity space, concentrations of stars with similar velocities - moving groups - can be clearly identified. Figure $6 \mathrm{~b}$ shows the wavelet-smoothed field (see, for example, Loktin \& Popova 2007) of peculiar stellar velocities. Moving groups appear to be of dynamical origin and are therefore convenient tools for the study of galactic dynamics.

Digital band filtering reveals numerous condensations of stars largely coincident with known stellar kinematic groups (Francis \& Anderson 2009; Antoja et al. 2008; Famaey, Siebert \& Jorissen 2008). The most complete dis- tribution of moving groups can be found in the paper of Zhao, Zhao \& Chen (2010) (the squares in Fig. 6b). Note that the moving group Stream 1 has been identified in none of the previous studies. Moving stellar groups are also well separated on the Lindblad diagram.

Figure 7 shows the HR-diagrams for moving groups (isochrones are adopted from Girardi et al. 2003). As is evident from the figure, the HR diagrams of moving groups located on the periphery of the peculiar velocity field are more similar to those of open clusters. This means that the age dispersion in moving groups located on the periphery on the diagram of the peculiar velocity field is not large.

\section{Conclusions}

1. The solar velocity components determined from the radial velocities and proper motions of stars are equal to $U_{0}=11.1 \pm 0.6 \mathrm{~km} \mathrm{~s}^{-1}, V_{0}=15.1 \pm 1.3 \mathrm{~km} \mathrm{~s}^{-1}$, $W_{0}=7.1 \mathrm{~km} \mathrm{~s}^{-1}$. Open cluster data yield the following velocity components: $U_{0}=9.4 \pm 0.2 \mathrm{~km} \mathrm{~s}^{-1}$, $V_{0}=11.0 \pm 0.5 \mathrm{~km} \mathrm{~s}^{-1}$. The differences between these results greatly exceed the error bars.

2. Nearby stars are not very suitable for the determination of solar velocity components because of the peculiarities in the velocity distribution. It is therefore more appropriate to choose objects from a larger galactic-disk volume. Kinematical groups make it impossible to unambiguously determine the velocity zero-point in the Galaxy.

3. The origin of moving groups is still unclear. The hypothesis that they are of purely dynamical origin (e.g., Famaey et al. 2008), which is based on the large age and metallicity scatter within each group, is not very justified, because group members are selected based on kinematic criteria exclusively.

Acknowledgements. This work was supported in part by the Russian federal task program "Research and operations on priority directions of development of the science and technology complex of Russia for 2007-2013” (contract 14.518.11.7064).

\section{References}

Antoja, T., Figueras, F., Fernandez, D., \& Torra, J. 2008, A\&A, 490, 135

Dehnen, W., \& Binney, J. 1998, MNRAS, 298, 387

Dias, W. S., Alessi, B. S., Moitinho, A., \& Lépine, J. R. D. 2002, A\&A, 389, 871

Famaey, B., Siebert, A., \& Jorissen, A. 2008, A\&A, 483, 453

Fehrenbach, Ch., Duflot, M., \& Burnage, R. 2001, A\&A, 369, 65

Francis, C., \& Anderson, E. 2009, New A, 14, 615

Girardi, L., Bertelli, G., Bressan, A., et al. 2003, Mem. Soc. Astron. Ital., 74, 474

Kharchenko, N. V., Scholz, R.-D., Piskunov, A. E., Röser, S., \& Schilbach, E. 2007, Astron. Nachr., 328, 889

Loktin, A. V., \& Popova, V. E. 2007, Astron. Reports, 51, 364

van Leeuwen, F. 2007, A\&A, 474, 653

Zhao, J., Zhao, G., \& Chen, Y. 2009, ApJ, 692, L113 

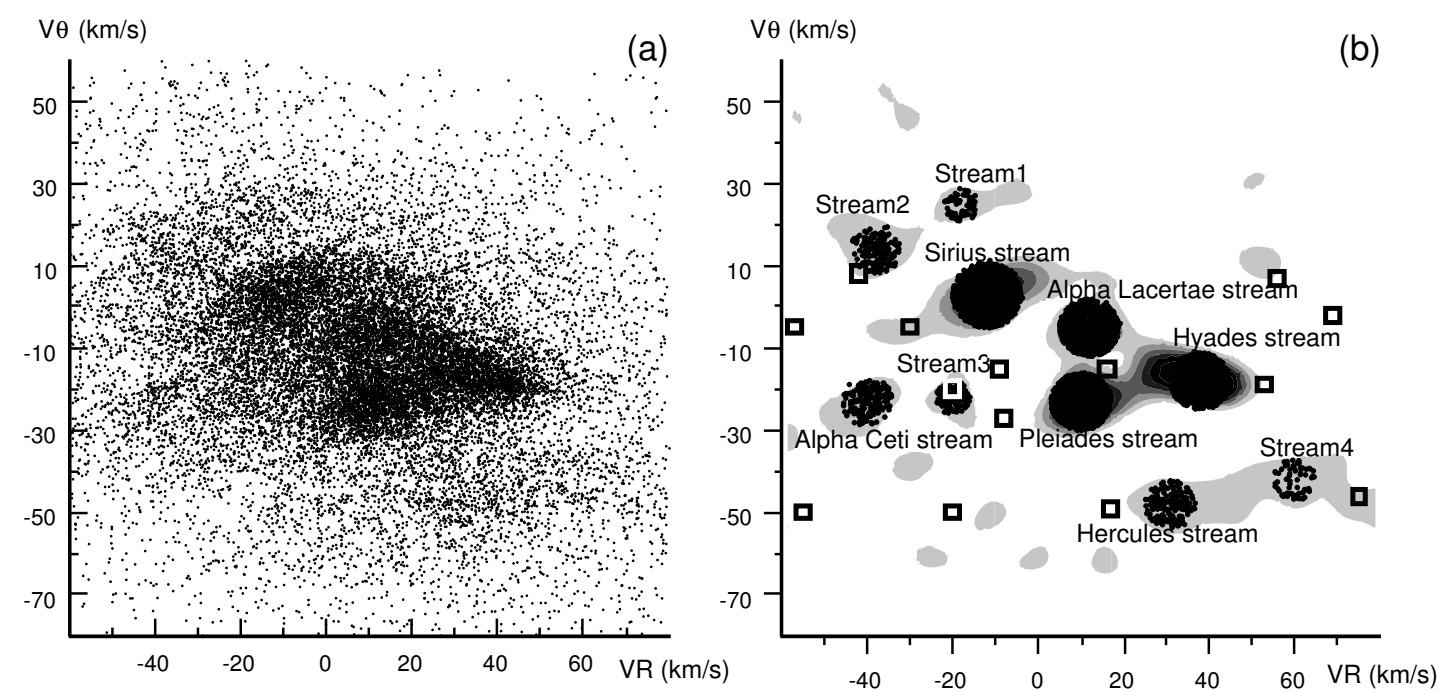

Fig. 6 The peculiar velocities of stars $(a)$ and wavelet-smoothed velocity field with moving groups identified $(b)$.

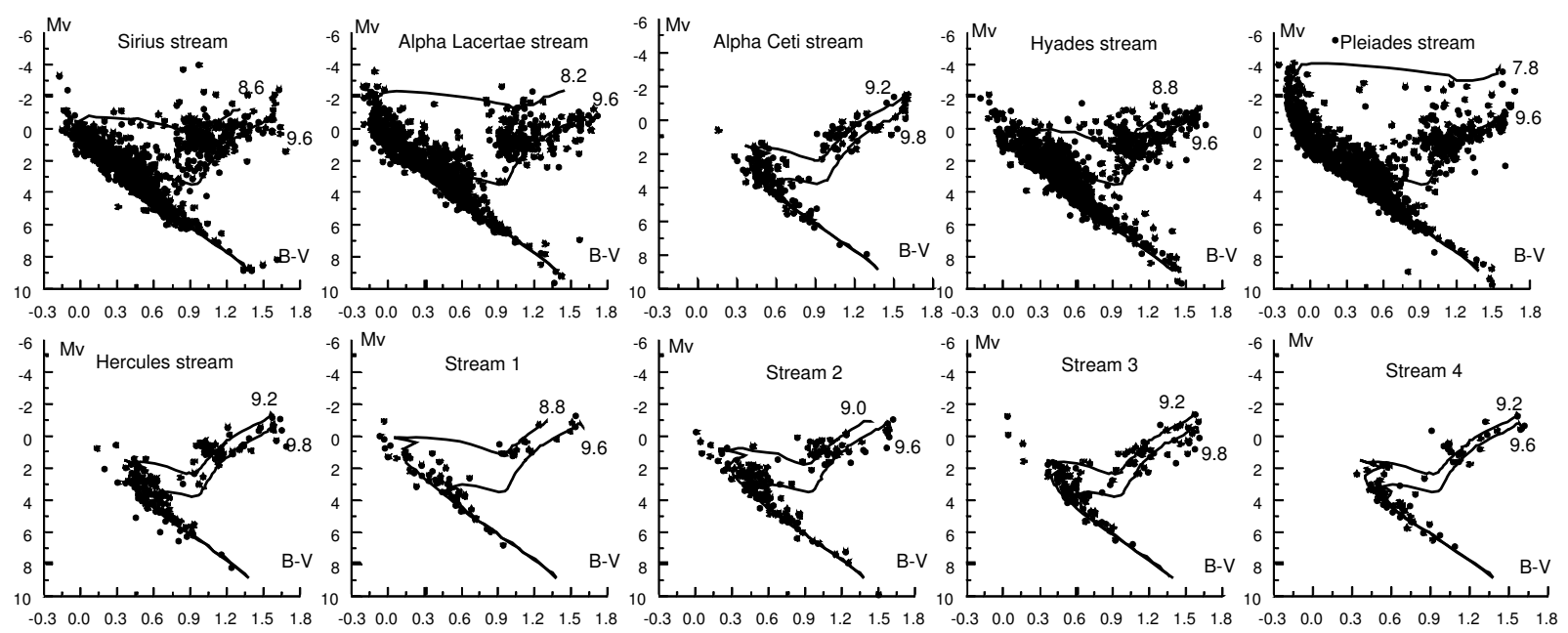

Fig. 7 HR-diagrams for moving groups (isochrones from Girardi et al. 2003). 